\title{
CONSERVACIONISMO EN ERAS DE ESPECIEÍSMO
}

\author{
Sandra Baquedano Jer $^{1}$
}

Recibido el 7 de mayo de 2014, aprobado el 21 de noviembre de 2014 y actualizado el 4 de mayo de 2015

DOI: 10.17151/luaz.2015.41.13

\section{RESUMEN}

Los alcances acumulativos de la acción humana, aunados al poderío tecno-científico, llevan hoy a que nuestra especie no solo se cuestione los límites de la capacidad que tiene la Tierra para asimilar y reciclar luego los desechos de la actividad humana, sino también los límites de nuestro especieísmo en la trama de la existencia. No ha sido la excepción, sino más bien la regla, que en una constante nuestra especie haya aumentado en población, sobreutilizando el entorno y malogrando un desarrollo que deteriora el medio ambiente cada vez más. El ser humano ha escrito la historia de la humanidad de forma especieísta, ensalzando su rol histórico que ha hecho obra contra el ser ecológico. Sin embargo, aisladas y frágiles treguas entre animales humanos y no humanos posibilitan cuestionarnos el sentido y significado filosófico del conservacionismo en eras del más crudo especieísmo.

\section{PALABRAS CLAVE}

Conservacionismo, especieísmo, responsabilidad, biodiversidad, daño ambiental, sustentabilidad, uso de recursos naturales.

\section{CONSERVATION IN SPECIESISM AGES}

\begin{abstract}
The cumulative scope of human action, combined with the techno-scientific power nowadays take our species not only to question the limits of the Earth capacity to assimilate and recycle the waste products of human activities, but also the limits of speciesism within the weave of existence. It has not been the exception but rather the rule, that our species has constantly increased its population, overusing the environment and ruining a development that deteriorates the environment increasingly. Human beings have written the history of humanity in a speciesist form, extolling their historic role which has made work against the ecological being. However, isolated and fragile truces between human and nonhuman animals allow questioning the meaning and philosophical significance of conservationism in an age of the crudest speciesism.
\end{abstract}

\section{KEY WORDS}

Conservation, speciesism, responsability, biodiversity, environmental damage, sustainability, natural resource use. 


\section{¿CONSERVACIONISMO IMPLICA SIEMPRE ANTIESPECIEÍSMO?}

El conservacionismo puede ser visto como una vía alternativa que intenta escindirse del camino trazado por el modelo económico imperante. En América Latina se muestra así como una vía que se opone y aleja de la óptica económica que explota y reifica el medio ambiente tasándolo únicamente como "recurso" natural. Pese a que el término proviene de conservar y con este verbo se puede designar en general una determinada disposición para mantener cuidadosamente los más variados procesos y fenómenos, el concepto suele asociarse en el marco ambiental tanto a la necesidad como a la importancia de conservar ecosistemas, especies y paisajes, entre otros, de la intervención humana que interfiere negativamente en tales espacios naturales. Así se busca cuidar y defender, por ejemplo, bellezas escénicas de impactos paisajísticos que destruyen la estética de una zona o enriquecer y proteger la biodiversidad de causas antropogénicas que pueden disminuirla, llevando incluso a la extinción de ciertas especies.

Si bien al rastrear la historia, se pueden encontrar diversas medidas conservacionistas, el movimiento actual puede ser considerado como un intento de frenar tanto el desarrollo de la industrialización como los alcances tecno-científicos modernos en ciertos espacios libres de dicha intervención. Aunque las problemáticas ecológicas actuales son globales, se intenta al menos resguardar algunas zonas de una intromisión antropogénica directa. ${ }^{2}$ Ello supone una dicotomía existente entre "gente" y "áreas protegidas", la cual es extremadamente amplia. De hecho, el concepto gente abarca desde los mismos conservacionistas hasta grupos de empresarios que únicamente buscan apropiarse y controlar los recursos naturales para su completa explotación. Cabe no obstante precisar que es entre los conservacionistas donde el diálogo entre ambas posturas implica una reflexión sobre cómo integrar en ecosistemas enteros el desarrollo sustentable. Entre ellos paulatinamente se ha ido difundiendo -en diverso grado y con propósitos distintos- la necesidad de proteger especies animales, lo cual lleva a enfrentar la interrogante de si puede concretarse un ideal conservacionista libre de toda práctica especieísta, pues la conservación de áreas protegidas no estaría exenta de esta problemática en cuanto ha implicado, por ejemplo, escoger paisajes y especies de belleza única para el gusto estético occidental en detrimento de pastos y animales considerados feos según los parámetros de selección (Monjeau, 2009). Así los escogidos pueden ser delfines o huemules en detrimento de insectos feos como baratas u otros arácnidos. Otra paradoja se puede visualizar en ciertos proyectos de restauración de ecosistemas donde se erradican masivamente animales que no pertenecen a especies autóctonas para recrear el ambiente original -por mor al ecosistema que se persigue restaurar y que se lo considera como un fin en sí- en detrimento de los miembros de otras especies no autóctonas. Queda en evidencia así que existen estos y otros múltiples criterios de selección que 
avivan una reflexión crítica en torno al sentido y significado del especieísmo. Si bien la popularización del término se la debemos a Peter Singer, su origen alberga una historia doble: la de su problemática y la acuñación del concepto mismo.

\section{ESPECIEÍSMO CON LOS ANIMALES NO HUMANOS}

El término speciesism proviene de especie, así como racismo proviene de raza. El especieísmo es respecto a la especie humana en su totalidad, lo que el racismo, a determinadas razas en particular. Este último se da a nivel de intraespecie; mientras que el primero supone el traspaso de ella. No se trata de una igualdad o semejanza sustancial, sino de un análogo referencial. El especieísmo se aplica a la creencia que afirma la superioridad de una especie en detrimento de las demás, y preconiza la separación de especies o grupos por segregación. Por ejemplo, en el caso del especieísmo contra los animales no humanos dentro de un hábitat artificial, como pueden ser los mataderos, los laboratorios, los zoológicos o los circos, por nombrar algunos. El especieísta niega que las demás especies sean dignas de poseer derechos, y no evita, sino que propicia, ya sea directa o indirectamente, el sufrimiento en seres no humanos.

Al respecto Gary Francione (2008, p. 26) denomina "esquizofrenia moral" a la doble realidad generada del hecho de que teóricamente miembros de la sociedad defiendan por un lado la necesidad de poner fin al sufrimiento animal; pero por otro lado ejerzan un trato o mantengan hábitos (alimentación o recreación) que atentan abiertamente en contra de la postura abstracta que ellos mismos defienden. Esta esquizofrenia moral es explicada por el hecho de que en el fondo los animales siguen siendo considerados como propiedades o mercancías del ser humano siendo rebajados al estatus de meras cosas. ¿Y si se les extendiera la posibilidad de dejar de serlo? ¿Podrían acercarse al estatus moral de personas? Según Francione (2008), lo moralmente relevante no radica en si un animal puede reconocerse o no en un espejo. Si así fuera se podría afirmar que los animales no humanos, al carecer de autoconciencia, no se les puede atribuir a sus dolores un significado ético, puesto que no existe un yo que dé continuidad a la experiencia psíquica. Esta afirmación podría a su vez resultar diáfana y sensata, sin embargo, no resulta lo mismo cuando se intenta, a partir de tal argumento, argüir que por ello los animales no humanos no sienten o padecen menos dolor y, por lo mismo, que no sufren ni merecen igual dignidad que el ser humano.

Si bien los requisitos para ser persona están asociados a cualidades características del Homo sapiens, el estatus moral en cambio ha sido atribuido a humanos que no albergan tales aspectos. En la misma línea ha de considerarse que hasta una empresa puede adoptar personalidad en términos jurídicos. En relación a esto cabe cuestionarse entonces qué es lo relevante en términos morales. Quienes piensan que reconocerse en un espejo es superior en términos morales a reconocerse por olores 
(Francione, 2008, p. 159), pueden ser más propensos a militar en una postura que niegue la posibilidad de dolor o sufrimiento en la línea de un speciesismus, y a afirmar que en tal caso la reacción ante un daño se trataría ante todo de meros reflejos defensivos, que carecen de una percepción consciente y sensitiva, pues esta no tendría lugar en la inmediatez de las respuestas instintivas.

Hasta ahora el uso explícito del término ha sido empleado únicamente en relación con los animales no humanos. Se trata, sin duda, de un sesgo que exige sondear y defender un extensionismo antiespecieísta. El especieísmo vegetal no ocurre de un modo tan evidente, sino ante todo cuando conscientes de una visión holística del mundo aparecen privilegiados material y superficialmente grupos de la especie humana a costa del profundo deterioro global del sistema existente entre las otras. El holismo mencionado contempla la concepción de que cada especie constituye un todo distinto de la suma de quienes la compongan (Baquedano, 2008, p. 88).

En similar contexto, Freya Mathews (1998) diferencia, por ejemplo, un holismo amplio que se distingue de las interpretaciones relacionales, tratando de esclarecer que si bien los individuos en una primera instancia se manifiestan teniendo una existencia ontológica, en un sentido más profundo esta resulta de la interacción que ellos mantengan entre sí, pues a partir de tal relación es que se define la individualidad de cada cual. Desde el holismo amplio el individuo puede ser considerado en su unión con el todo. Por consiguiente, la individualidad pierde en el fondo su significado ontológico cuando se la analiza desde un nivel más profundo.

Andrea Speranza (2006, p. 43) en su intento de esclarecer el sentido y significado de la Gestalt en la ecología profunda de Naess, considera factible concebir la naturaleza de dos maneras:

[...] una expansión de uno mismo, de modo que defenderla es, en cierto sentido, defender el propio ser, o bien podemos entendernos a nosotros mismos como una expresión de la naturaleza y, así, los intereses de ésta son, de alguna forma, nuestros propios intereses.

Tales concepciones de la naturaleza permiten pensar una realidad que se articula en un campo relacional posibilitando a cada especie adquirir un sentido y significado a partir de las interacciones con el resto de las especies y la biosfera en general. Sin embargo, desde esta perspectiva la especie podría transformarse a su vez en la apariencia de un ser más profundo en cuanto constituye una totalidad integrada.

El análisis de lo ambiental desde una perspectiva integradora permite sondear el hecho de que la naturaleza se haya dañado a sí misma. La relación con el medio ambiente natural no es la de un intelecto totalmente inmaterial que se confronta a través de un dualismo con objetos materiales inertes, los cuales no influyen en un sentir o padecer mayor. El medio ambiente natural es 
precisamente la relación que se establece con él. Análogamente a la fusión sujeto-objeto de la mecánica cuántica, el estudio de la naturaleza es a su vez el del ser sí mismo, pero este sí mismo no está solo dentro de cada cual, sino que forma parte de una relación mayor. Algo que Berman (2007, p. 16) denomina "conciencia participativa", es decir, que activa y fuertemente lo conforma, identificándolo con la naturaleza. Si se actúa de tal forma no se puede llegar a comprenderla de un modo distinto aunque exista un afán en hacerlo. La mente y el cuerpo, lo interno y lo externo, sujeto y objeto, son aspectos de un mismo proceso. Las totalidades son capaces de sufrir un padecimiento que las partes no padecen necesariamente. Que la decadencia ecológica que se ha desencadenado en la Tierra no solo ponga en peligro la existencia de otras especies vegetales y animales, sino también la misma especie humana, deja en evidencia el carácter especieísta de la relación que el ser humano mantiene con el resto de las formas de existencia, quedando en evidencia así, la necesidad de buscar vías alternativas que consoliden la posibilidad de una relación antiespecieísta o menos especieísta.

\section{RASTREANDO LOS ORÍGENES DEL ESPECIEÍSMO CONTRA LOS ANIMALES}

Cronológicamente, el Homo habilis es el primer homo. Su aparición data de aproximadamente 1,8 millones de años y recibe su nombre debido al manejo y elaboración de los útiles de piedra. Hoy se sostiene que convivió con diferentes tipos de Australopithecus. Estos "simios del sur" son probablemente los antecesores inmediatos del homo. Se cree que aparecieron hace más de tres millones de años y habitaban en las zonas tropicales de África (Leakey, 1981, p. 57). Se sabe que se alimentaban de frutas y hojas, que se desplazaban de manera bípeda y que el tamaño de su cerebro no difería en mucho al de los grandes simios actuales. Se sostiene que fue precisamente la presión que ejerció la especie del homo sobre la del Australopithecus aquello que pudo haber incidido sustancialmente en su desaparición. Un escenario probable es que ambos estilos de vida, si bien fueron diferentes, se parecieron lo suficiente como para que se intensificara una competencia relativa a la comida y el espacio que se fue incrementando a medida que el homo afirmara su hegemonía en el espacio que habitara (Leakey, 1981, p. 97). Sin embargo, a pesar de la aparente superioridad técnica del Homo habilis, que habría incidido en la extinción de nuestros antecesores los Australopithecus, no se podría sostener que se habría incurrido en lo sustancial en un especieísmo de parte del Homo habilis, debido a que las similitudes anatómicas que se establecen entre ambos eran considerables. Es decir, la facultad para realizar operaciones conceptuales y simbólicas complejas como por ejemplo el empleo de sistemas lingüísticos sofisticados, las capacidades de introspección, especulación autocrítica y razonamiento abstracto, no son rasgos característicos ni del Homo habilis y mucho menos del simio del sur -el Australopithecus-, sino sobre todo son consideradas facultades atribuibles al Homo sapiens; de ahí su adjetivo: sapiens. 
Según las hipótesis tradicionales, el Homo habilis evolucionó hacia el Homo erectus, hace unos 1,5 millones de años y luego este comenzó a ser reemplazado por formas arcaicas de Homo sapiens entre hace 400 y 250 mil años. Desde hace unos 30 mil años, el Homo sapiens es la única especie sobreviviente del homo. El adjetivo sapiens alude al rasgo biológico más característico: sapiens significa "sabio" o "capaz de conocer", y se refiere a la consideración del ser humano como "animal racional", a diferencia de todas las otras especies. Paradójicamente, la estrechez sapiente del homo ha tergiversado y confundido a este a diferencia por en detrimento o en contra del resto de las especies. El hecho es que el origen y delimitación del concepto "especieísmo" proviene de Richard Ryder, cuando en 1970 acuñó el término en su artículo "Experiments on Animals" en vista de denunciar los crueles experimentos que se realizaban en los laboratorios con animales. Ahí condenó la postura contradictoria en la que solían incurrir los científicos de su época, puesto que muchos de ellos aceptaban la teoría de la evolución de Darwin, la cual supone un continuo biológico; es decir, aceptaban por un lado la existencia de una similitud sustancial entre un chimpancé y un ser humano (el código del ADN entre ambos guarda similitudes notables); pero, por otro lado, establecían en la práctica una distinción moral radical a la hora de experimentar con ellos. ¿Cómo entender esta conducta contradictoria? Utilizando una casuística imaginaria desde Schopenhauer, Ryder, Singer o Jonas, se podría conjeturar respectivamente: actúa en vista de satisfacer intelectualmente las necesidades insaciables de su yo volente, experimenta en aras de aumentar su conocimiento y beneficiar su propia especie, obra sin acatar la idea de un continuo evolutivo fisiobiológicosocial o actúa simplemente según la tendencia utópica del Homo faber con la disposición a utilizar a las demás especies que ahora viven como simple medio para una determinada meta o apartarlas como un obstáculo para ella.

Sufrir es un fenómeno tan complejo y profundo que no puede ser comparado en padecimiento ni en intensidad con otra capacidad. Bentham (1970) sostiene que el estatus que adquiere la capacidad de sufrimiento le otorga a todos los sintientes una dimensión común. Ahora bien, esta postura es considerada utilitarista reformista en la medida en que el sufrimiento se pone en relación con los intereses de todos los seres con capacidad de sufrimiento o goce. Tanto lo uno como lo otro son requisitos para tener cualquier tipo de interés. Cuando posteriormente se abogue por los derechos de los animales, tal exigencia se desprenderá sustancialmente de la posibilidad de que ellos puedan sentir dolor. Ahora bien, existen clásicas posturas como las que defiende Hans Jonas (1979) las cuales sostienen que para tener derechos, un ser debe ser autónomo, poseer sentido de justicia y discernimiento; es decir, ser capaz de respetar los derechos de los otros. Se aboga al respecto por una Ética de la responsabilidad, donde se reconoce que la única superioridad de la especie humana consiste en su capacidad para ser responsable por el resto de los seres vivos que habitan el planeta, es decir, su "dignidad ontológica" -dice el que fue en su juventud discípulo de Heidegger- estriba en ser el único ser que puede ser custodio de ellas. De ahí que primaria sea la 
responsabilidad intrahumana, en cuanto el ser humano es sujeto de responsabilidad de otros seres (Jonas, 1979, pp. 84-5).

Según Bentham (1970, cap. 17) y Singer (1999, cap. 1) el único límite defendible a la hora de preocuparnos por los intereses de los demás es el de la sensibilidad. Esta última remite, no solo a la capacidad de sufrir, sino también de disfrutar.

Hasta ahora los animales no humanos no han podido defenderse del especieísmo contra ellos y la complicidad que abala tal discriminación. La tesis de que los animales no sufren fue sostenida implícitamente por Descartes cuando declara que los animales son autómatas. El dualismo cartesiano concibe en lo esencial únicamente dos realidades: pensamiento y extensión. De estas se desprenden dos principios de movimientos: el primero es incorpóreo, puesto que concierne al espíritu, alma o sustancia pensante; el segundo es enteramente mecánico y corpóreo. Este último concierne -según Descartesprecisamente a los animales. Así los concibe como autómatas, explicando todos sus movimientos a través de principios mecánicos. Gómez Pereira en esa misma época expuso en su obra principal, la Antoniana Margarita, que los animales carecen de facultades sensibles, sustentada en la tesis de que los animales carecen de alma ${ }^{3}$. Brutus sensa carere, puesto que si poseyeran sensibilidad habría que atribuirles una inteligencia como la del ser humano. A Gómez Pereira le resultaría imposible atribuirles entendimiento -imposibilidad, tanto empírica, como asegurada, por su concepción filosófica y teológica-; negaría su potencial sensitivo en la medida en que la sensibilidad externa requiere el sentido común que es consecuencia del uso de los sentidos. A este hecho le sigue la afirmación de que el movimiento de los animales es de índole mecánica y no espontánea.

El especieísta niega la capacidad que tienen los animales para sentir dolor, aun cuando sea posible observar sus comportamientos: sacudidas, contorsiones faciales, gemidos, chillidos $u$ otros sonidos; en fin, diversos intentos de evitar la fuente de dolor y retirar bruscamente determinados miembros cuando han sido lesionados, además de cambios fisiológicos ante el daño en potencia que se percibe como aumento inicial de la presión de sangre, transpiración, aumento de las pulsaciones, dilatación de las pupilas, hasta el extremo de desencadenarse en el animal manifestaciones contrarias cuando el agente agresor lo ha dañado severamente (Singer, 1999).

Si bien son innegables estas reacciones, que provienen de seres cuyos sistemas nerviosos son casi idénticos fisiológicamente con un origen y función evolutiva común-, el especieísta niega, no obstante, que puedan funcionar de manera similar ante el dolor. $\mathrm{Y}$ todo esto aun cuando se considere que tanto ese dolor como sufrimiento no son efectivamente comprobables del mismo modo que en la especie de los sapiens.

Desde una perspectiva biológica, se suele definir el dolor como una experiencia sensorial y emocional desagradable, que se 
encuentra en relación con algún tipo de lesión tisular real o un daño potencial. La reacción ante tal daño es lo que estimula precisamente un actuar. Por un lado, si se considera el dolor desde un puro plano fisiológico, como mecanismo comprensible únicamente, mediante razones suficientes, entonces solo duraría y actuaría durante el tiempo y con la intensidad que exige su función biológica. En este caso una persona que padeciese una enfermedad crónica o irreversible, no debería sentir ya ningún dolor, al no cumplir en la práctica ninguna función. Por otro lado, si se considera solo la experiencia o el daño, comprobados empíricamente tras razones suficientes, y no dentro de la vivencia o la abstracción que una persona emprende de ella, podrían ser consideradas las alucinaciones de un enfermo psiquiátrico agudo o el umbral de abstracción que él mismo emprenda de ella, como exclusivas señales o consecuencias de una enfermedad, y no como fuente veraz de padecimiento, que refleja su verdadera posición existencial.

Cuando los mecanismos defensivos no ayudan a canalizar el dolor y se vuelven contra quien lo padece, se genera una suerte de atascamiento que se identifica con el término sufrimiento antes que dolor. Spaemann (1977, p. 118) ayuda en este sentido a comprender la connotación referida: "El término alemán 'sufrimiento' tiene, de manera análoga a sus términos correspondientes en otras lenguas, un doble sentido. Significa tristeza (infelicidad, desagrado...), y también sencillamente pasividad (en el sentido de passibilitas), o, por decirlo a la moda, frustración".

A partir de un cierto grado de intensidad, el dolor como tal pasa a ser sufrimiento. El paso de uno a otro, ocurre cuando se anula por la profundidad del padecimiento todo motivo positivo o negativo del futuro; cuando se pierde la esperanza de alivio real y se estanca con ello la canalización que los aleja de su intensidad. Aquí entonces ya no cabe hablar de dolor, sino de sufrimiento, en cuanto comprende la totalidad de un estado físico y mental.

El dolor al ser más específico que el sufrimiento tiene claramente una utilidad biológica, ya que debiera aumentar la posibilidad de supervivencia de la especie, y permitir evitar las fuentes de daño $y$, por consiguiente, de sufrimiento con independencia de la raza, la especie o el género de un ser vivo (Singer, 1999, p. 53).

\section{¿SELECCIÓN NATURAL O ANTROPOGÉNICA DE LAS ESPECIES?}

Masificado y agudizado el especieísmo a través del desarrollo técnico-industrial y la ideología económica imperante, el ser humano ha explotado y hecho sistemáticamente desaparecer plantas y animales silvestres. Al tener el poder de extinguirlas de la naturaleza, surge también como referente la responsabilidad para protegerlas en un ambiente que permita su conservación natural. Al respecto, algunos centran su preocupación en la supervivencia de las especies, mientras que otros ponen énfasis 
en la necesidad de terminar con la crueldad hacia los animales, cuestionando severamente el especieísmo, pues rechazan la ilusa creencia en la superioridad del Homo sapiens e integran a su causa a movimientos que luchan por la defensa de los animales. De cualquier forma, en ambos casos subyace una crítica a la especie humana por ser la única que se concibe como fin en sí, es decir, como la única en el mundo que no acepta bajo ninguna circunstancia ser tratada solamente como medio, mientras que todo el resto queda disponible a su servicio. En relación a este hecho Hans Jonas (1979, p. 184) sostiene, por ejemplo, que cada ser vivo es su propio fin y no está necesitado de ulterior justificación. Desde tal perspectiva el ser humano no es aventajado con respecto a las demás especies, pues su "dignidad ontológica" estriba únicamente en que solo él puede ser también responsable por ellas. A raíz de tal capacidad se considera que el prototipo de la responsabilidad es la intrahumana. Tal relación no es recíproca y alude más a una unilateralidad. En este caso, remite a la responsabilidad del ser humano por áreas ricas en ecosistemas frágiles que han de ser protegidos en cuanto se encuentran en una situación indefensa, de vulnerabilidad y desventaja. En este contexto también implicaría, por ejemplo, prevenir o aliviar el padecimiento de animales no humanos en situación de sufrimiento, es decir, procurarles una asistencia cuando lo requieran si es que está en poder del ser humano hacerlo. Si bien la relación de responsabilidad intrahumana es en sí unilateral puede, sin embargo, ser reversible e incluye la posibilidad de darse una reciprocidad.

John Maxwell Coetzee (2001) critica la división aristotélica entre dioses, humanos y bestias, replanteando implícitamente el especieísmo desde la perspectiva de los derechos de los animales y extrapolándolo a la relación del ser humano con la naturaleza. Según detalla Singer (1999, pp. 232-259), esta forma de discriminación fue legitimada por la religión judeo-cristiana, la cual sitúa al ser humano como el centro de la creación. A esta cosmovisión le sucedió la visión heliocéntrica del universo, la cual se impuso a pesar de la reserva y desconfianza de la Iglesia Católica. En épocas posteriores la teoría de la evolución de las especies resituó al ser humano como uno más en la trama de la vida. El efecto aquí fue doble: por un lado se acentuó el distanciamiento entre el saber científico y la doctrina eclesiástica, dejando, por otro lado, el darwinismo en evidencia la vulnerabilidad de toda especie (incluyendo la humana) ante un desequilibrio ambiental. Esta fragilidad permite explicar que El origen de las especies no solo sirviera de preludio en la reflexión relativa a la problemática de la extinción -en los marcos de la selección natural-, sino también que ayudara a generar poco a poco conciencia del potencial destructivo del Homo sapiens.

Hoy en día es posible darse cuenta que de modo similar a como son admitidas determinadas conductas autolesivas en otras especies y ecosistemas, la historia mostraría que también existen algunas tendencias autodestructivas (sistemas bélicos, tecnológicos, económicos y en definitiva antiecológicos) que inciden en las bases sistemáticas que han sostenido la vida en el planeta pudiendo desencadenar variantes radicales de 
autodestrucción colectiva, cuyo anverso podría ser el nacimiento de nuevas formas de vida.

Como un modo de compensar tal tendencia aparecen hoy las áreas protegidas en aras de contener el desmesurado deterioro de la superficie terrestre derivado de actividades extractivas. ${ }^{4}$ Sin embargo, la historia muestra que esta conservación no ha estado exenta de ciertas problemáticas relativas al especieísmo, como puede ser entre diversas variantes, la selección de ciertas especies en detrimento de otras. Monjeau (2009), por ejemplo, vincula fuertemente la defensa de la belleza natural con el uso de la violencia. Considérese que la primera connotación del término belleza que confiere la RAE es el de la "propiedad de las cosas que hace amarlas, infundiendo en nosotros deleite espiritual. Esta propiedad existe en la naturaleza y en las obras literarias y artísticas". Cabe cuestionarse luego ¿qué propiedades existen en la naturaleza que nos hacen amar sus elementos o seres infundiendo deleite espiritual? Parece imposible obtener una sola respuesta universal, pues la belleza es un fenómeno cultural, histórico y psicológico impregnado de subjetividad. No obstante, se pueden encontrar márgenes relativos a determinadas estructuras y aspectos estéticos que existen en desmedro de otros. A través de tales patrones se suele discernir luego entre lo bello y lo feo, pues se traducen en ciertas estructuras que generan fenómenos atingentes al llamado placer o deleite visual. Los primeros tratados al respecto se remontan a los antiguos griegos. Platón y Aristóteles describen profundos aspectos sobre los componentes de la armonía, el sentido y significado de la simetría, orden y proporción como características de la belleza. A tales planteamientos le suceden dos milenios de producción en las más variadas esferas del arte y hace un par de siglos se ha incorporado a la misma naturaleza como trasfondo.

Muchos parques nacionales surgieron en aras de preservar espectaculares bellezas escénicas, acompañadas de hermosos animales que la gente suele pagar por ver, independientemente de lo que se haga o no para defenderlos de su extinción. Pese a ello, gracias a los sistemas de parques nacionales se ha conseguido preservar la biodiversidad y la belleza de parajes únicos en el planeta de la devastación global.

Las promesas del progreso, el éxito y el crecimiento de las grandes urbes se utilizaron para justificar la destrucción de vastas zonas rurales. Sin embargo, la pérdida de la belleza tanto en el campo como en la urbe convierte toda promesa en una apocalíptica amenaza que avanza visiblemente junto al especieísmo hacia una empinada decadencia ambiental.

Si bien la búsqueda de la belleza ha resultado ser en la práctica un elemento que a veces ha favorecido diversas iniciativas ecológicas -entre ellas los procesos de conservación- esta tendencia no supera del todo los diversos grados y modalidades del especieísmo. Pese a ello, sería un absurdo deslegitimar por tal razón los proyectos y las iniciativas de proteger las áreas de conservación que surgieron a partir de un determinado encantamiento estético. Únicamente se intenta dejar de 
manifiesto cuan profunda y compleja resulta ser la problemática del especieísmo. En otras palabras: si bien hombres y mujeres al ser cautivados por parajes naturales de una belleza única, pueden sensibilizarse y realizar, por ejemplo, millonarias donaciones a fin de proteger diversas reservas que provocan tal embelesamiento o involucrarse altruistamente en causas que promueven la defensa de áreas prístinas, el problema del especieísmo no se extingue del todo.

Cabe precisar que la justificación de conservar áreas protegidas -incluyendo por ejemplo los parques nacionales- no descansa en meras razones estéticas pues para los ecosistemas son imprescindibles vastos espacios naturales a fin de salvaguardar su equilibrio. Esto exige preservar la biodiversidad en general con todas sus rarezas, como a su vez regular el agua, el aire, los suelos o el mantenimiento de la estabilidad en las pendientes, todo lo cual es llevado a cabo por razones que trascienden la esfera estética. De hecho, en tales procesos se esclarece la importancia de crear áreas protegidas en zonas que no son consideradas necesariamente bellas, lo cual deja de manifiesto a su vez que el cuidado y valor por la biodiversidad está más cercano y en camino a tender a un móvil antiespecieísta aun cuando no se pueda superar del todo el especieísmo frente al cual se combate.

\section{CONSERVACIONISMO COMO CONCIENCIA DE ESPECIE: EN BÚSQUEDA DE UN MÓVIL ANTIESPECIEÍSTA}

Atenerse a la posible capacidad de sufrir en las especies no humanas no cambia necesariamente el antropocentrismo epistémico, pues el especieísmo implica la percepción del mundo derivada de la pertenencia y conducta discriminatoria de la especie humana con el resto de las formas de vida. Sin embargo, posibilita sondear diversos grados de conservacionismo para hacerle frente no como un asunto meramente biológico sino esencialmente filosófico. "La ética biocéntrica plantea que la biodiversidad tiene un valor intrínseco, por eso se privilegia a las especies biológicas y se excluye al ser humano de muchas áreas naturales (Rozzi, 2001; Turner et al., 2001). En cambio, la perspectiva ecocéntrica estima que los seres humanos son considerados como componentes de los ecosistemas y otorga un valor moral a las especies que constituyen las comunidades biológicas, incluyendo al Homo sapiens. Esa ética fue cobrando fuerza desde mediados del siglo XX con la ética de la Tierra formulada por Aldo Leopold (1949), quien invita a establecer una nueva forma de relación con la naturaleza: en lugar de ver al planeta como un bien que nos pertenece, se debería pensar en la Tierra como una comunidad a la que pertenecemos (Kinne, 1997; Rozzi, 2001; Turner et al., 2001; Kostas et al., 2003; Fernández-Manzanal et al., 2007; Shepardson et al., 2007). ${ }^{5}$ Al respecto, Michael Soulé, Paul Ehrlich y Jared Diamond comenzaron a desarrollar como disciplina formal la biología de la conservación, siendo fundada en 1985 la respectiva Sociedad para la Biología de la Conservación, la cual tiene como misión 
investigar, por una parte, el modo en que el ser humano influye sobre la biodiversidad $y$, por otra, desarrollar estrategias preventivas en aras de evitar la extinción de las especies (Corchera y Ponce de León, 2004, p. 204)".

A su vez la Ecología profunda es una filosofía que nace precisamente de la urgencia de transformar la adversa relación que ha dispuesto el ser humano con el entorno. Desde la perspectiva de un ecocentrismo, enfatiza la existencia de infinitas interrelaciones entre los diversos sistemas de la vida y apela a la identificación con todas las especies, albergando a la ecósfera, en vista de trascender el principium individuationis y develar el entramado común con todos los seres.

Actualmente la humanidad es testigo de la aparición de fenómenos acumulativos y globales como la sobrecarga del planeta derivada de la explosión demográfica y el sobreconsumo, el cambio climático, la pérdida de la biodiversidad, el adelgazamiento de la capa de ozono, la contaminación de los océanos, aunado todo ello a un sinfín de otros más localizados como la deforestación, desertificación, contaminación de tierras, ríos, lagos, agotamiento de napas subterráneas, etc. Si bien el fenómeno de la extinción de ciertas especies forma parte de un proceso natural, en la actualidad acontece masiva y aceleradamente como resultado de estas y otras causas antropogénicas (Stuart, 2000).

A diferencia del resto de las especies, la humana es la única responsable de la condición actual del planeta y la única que en su calidad de "sapiens" pudo haber evitado dañar el medio ambiente, lo cual sin duda ha marcado especieístamente su rol en la historia de la Tierra. Los problemas ambientales -que ponen hoy en riesgo la vida en el planeta- dejan cada vez más en evidencia la necesidad de sondear crítica y reflexivamente una determinada conciencia como especie a partir de una identidad que trascienda el género, la raza, el origen, ideología y cultura de una persona. Una conciencia que es a su vez autoconsciente de cuan profunda es la problemática del especieísmo a la hora de enfrentar el actual daño ambiental y de cuan arraigado está tal forma de discriminación aun cuando se abogue por prácticas conservacionistas que intenten combatirlo.

\section{REFERENCIAS}

- Baquedano, S. (2008). Sensibilidad y responsabilidad socioambiental. Un ensayo de pesimismo autocrítico. La Habana: Editorial Acuario.

- Bentham, J. (1970). Introduction to the Principles of Morals and Legislation. London: The Athlone Press.

- Berman, M. (2007). El reencantamiento del mundo. Trad. Sally Bendersky y Francisco Hunneus. Santiago: Editorial Cuatro Vientos. 
- Bruner, A., Gullison, R., Rice, R. y Da Fonseca, G. (2001). Effectiveness of parks in protecting tropical biodiversity. Science, 291, 125-128.

- Coetzee, J.M. (2001). La vida de los animales. Barcelona Ciudad de México: Grijalbo Mondadori.

- Corchera, P. y Ponce de León, L. (2004). Tendencias de los movimientos conservacionistas y el surgimiento de la Eco-Ética. Sociológica, 19(56), 199-211.

- De Castro, A., Cruz, J.L. y Ruiz-Montoya, L. (2009). Educar con ética y valores ambientales para conservar la naturaleza. Convergencia. Revista de Ciencias Sociales, 16(50), 353-382.

- Fernández-Manzanal, R. et al. (2007). Evaluation of environmental attitudes: Analysis and results of a scale applied to university students. Science Education, 91(6), 988-1009.

- Francione, G. (2008). Animals as Persons: Essays on The Abolition of Animal Exploitation. New York: Columbia University Press.

- Jonas, H. (1979). Das Prinzip der Verantwortung. Frankfurt am Main: Editorial Suhrkamp.

- Kinne, O. (1997). Ethics and ecoethics, Marine Ecology Progress, Germany: International Ecology Institute.

- Kostas, J. et al. (2003). Images of nature in Greek primary school text books. Science Education, 88(1), 72-89.

- Leakey, R. (1981). La formación de la humanidad. "La génesis de la agresividad en el hombre". Barcelona: Editorial Serbal.

- Leopold, A. (1949). A Sand County Almanac. New York: Oxford.

- Mathews, F. (1998). Ecological World Views. Encyclopedia of Philosophy. Londres-New York: Routledge.

- Monjeau, A. (2009). Guns \& Roses: violencia y belleza en la relación hombre-naturaleza. Cuadernos de Ética, 24(37).

- Rozzi, R. (2001). Ética Ambiental: raíces y ramas latinoamericanas en fundamentos de conservación biológica. En Primack, R. et al. (Comps.), Perspectivas Latinoamericanas. México: Fondo de Cultura Económica.

- Shepardson, D. et al. (2007). Students' mental models of the environment. Journal of Research in Science Teaching, 44(2), 327-348.

- Singer, P. (1999). Liberación animal. Madrid: Editorial Trotta.

- Spaemann, R. (1977). Einsprüche, christliche Reden. "Über den Sinn des Leidens" (Sobre el sentido del dolor). Einsiedeln: Johannes Verlag.

- Speranza, A. (2006). Ecología profunda y autorrealización. Introducción a la filosofía ecológica de Arne Naess. Buenos Aires: Editorial Biblos.

- Stuart, F. et al. (2000). Consequences of changing biodiversity, Nature, Insight Review Articles, mayo, vol. 405, Estados Unidos: MacMillan.

- Turner, N. et al. (2001). Éticas ambientales y conservación en los extremos de América. Fundamentos de Conservación biológica. En Primack, R. et al. (Comps.), Perspectivas Latinoamericanas. México: Fondo de Cultura Económica.

1. Licenciada en Filosofía por la Universidad de Chile. Magíster en Filosofía por la Universidad de Chile. Doctora en Filosofía por la 
Universidad de Leipzig. Profesora Asistente en el Departamento de Filosofía de la Universidad de Chile. sandrabaquedano@u.uchile.cl

Este artículo se enmarca dentro del proyecto Fondecyt, número 1120730 y 1140721.

2. Cf. Proyecto Diccionario del pensamiento alternativo II. Adrian Monjeau y Herminia Solari.

3. No fue posible encontrar el original de la obra Antoniana Margarita, opus nempe physicis, medicis, ac theologis non minus utile, quam necessarium (Madrid 1749). Cf. José Ferrater Mora. (1999). Diccionario de filosofía. Tomo II. Barcelona: Editorial Ariel. p. 1486.

4. Cf. Bruner et al. (2001).

5. De Castro, Cruz y Ruiz-Montoya (2009, p. 357).

Para citar este artículo: Baquedano Jer, S. (2015). Conservacionismo en eras de especieísmo. Revista Luna Azul, 41, 240-253. Recuperado de http://lunazul.ucaldas.edu.co/index.php?option=content\&tas k=view\&id=1064 